\title{
Certain Bio-Cognitive and Quantum Views of Depression
}

\author{
James Paul Pandarakalam
}

Department of Psychiatry, North West Boroughs healthcare NHS Foundation Trust, Hollins Park Hospital, Warrington, United Kingdom

\section{Email address:}

jpandarak@hotmail.co.uk, james.pandarakalam@nwbh.nhs.uk

\section{To cite this article:}

James Paul Pandarakalam. Certain Bio-Cognitive and Quantum Views of Depression. American Journal of Psychiatry and Neuroscience. Vol. 6, No. 2, 2018, pp. 33-45. doi: 10.11648/j.ajpn.20180602.12

Received: April 30, 2018; Accepted: May 30, 2018; Published: July 4, 2018

\begin{abstract}
Depression has been considered essentially a psycho-bio, social condition. In this paper, a hypnotic/quantum approach to understanding depression is attempted and parallel or co-thinking is designated as quantum thinking, one of the main sources of depressive cognitions. It is the flexibility of the quantum brain that accounts for cognitive distortions. Hypnosis may involve a spectrum of quantum consciousness and has great research value in uncovering non-biological depression. Like many of the alternate states of consciousness, hypnosis may be a neuro-quantum state. In the earlier part of the 2oth century, Frederic Myers recognised that the middle realm of the unconscious mind is the "hypnotic stratum" and is associated with deep hypnosis. The unconscious is increasingly allied with the quantum and it is time to revise the concept of the Freudian unconscious accordingly. The co-existence of a quantum mechanical body and brain is all the time more recognised now in parasciences. If consciousness is transmitted through rather than actually produced by the brain, depression may have a downward causation rather than an upward causation arguable in biological disorders like schizophrenia and bipolar disorder. Consciousness-based healing is warranted in the treatment of depression. Whether consciousness is the primary generator of clinical depression or it only contributes to the genesis of depression will continue to be a matter for debate in the foreseeable future.
\end{abstract}

Keywords: The Unconscious, Depressive Cognition, Hypnotherapy, Quantum, Biological Correlates, Cognitive Therapy, Antidepressants

\section{Introduction}

Depression has both biological and cognitive aspects, and which of these two is dominant is a matter of controversy. It is a conscious experience that has subjective aspects which pose fundamental questions about the nature of consciousness. There is no absolute cause for depression and therefore that no single answer should be sought for its amelioration. A multidimensional approach is the most desirable one. Given the complexity and minute scale of neuronal and mental phenomena, it is suggestive to reconsider these issues in the light of newer insights from quantum physics and it is high time for psychiatric profession to integrate non-classical ideas from other arenas of science in their research [1].

The multidimensional approach proposed in this paper excludes bipolar depression and secondary depression from the general discussion on non-biological depression (NBD).
The general term 'depression' is used here to denote cognitive depression that stems from non-biological causes but has biological correlates. A combined model incorporating cognitive, hypnotherapeutic and quantum views offers a more focused picture of cognitive depression, and the addition of psychodynamic and para-psychodynamic approaches to this dual model explains some of the processes involved in the pathogenesis of depression.

\section{Biological Correlates}

In most cases, depression has biological correlates but not necessarily biological causes. Emotional wounds may cause alterations in neurotransmitter levels or receptor sites. The powerful, resonant events of life may interact with brain chemistry, provoking shifts in mood states, but nobody can conclusively assert where biological factors intersect with non-biological ones. The biochemical basis of depression is usually analysed from genetic and biochemical perspectives. 
Family, twin and adoption studies have shown that there is a significant genetic component in depressive disorders, and such a concept has significance in relation to the severity of the illness. After all, material body is the software of the psychic apparatus.

As Pylkkannen [2] opined that the basic problem in quantum mechanics is unravelling the relationship between consciousness and the material world, the biggest riddle is establishing where biological factors and non-biological factors of depression intersect. The biological correlates of depression linking the "frontiers of brain" with the quantum may be explained using the interactome hypothesis proposed by Cocchi and his colleagues. Their novel hypothesis explains critical points of potential linkage between platelets and depression, including serotonin and membrane platelet fatty acids, in relation to the cytoskeletal quantum-nanowire network [3]. Platelets are hailed as 'the circulating neurone or the ambassadors of brain'. Evidences are now accumulating to prove that messenger molecules translate brain's most minute activity into chemicals that carry the same activity to every cell in the body and due to the presence of the myriad receptor sites on the outer membrane of each cell, the qualities once ascribed to the brain are now proven to be shared with the rest of the body [4]. In fact, the whole body presents with the biological correlates of depression. The traditional view that mind is fixed only in the brain may be false; mind may be rooted in the brain rather as a tree is rooted in the ground, with peripheral roots spreading all over the physical body. It is interesting to note that the heart transplant operations of the 1960s demonstrated the inadequacy of the belief that the heart is occupied by the mind.

Consciousness may have a physical, unphysical and even non-physical components, but it is bound to have a neural beginning or ending at the level of the brain. If consciousness is the musician, brain is the musical instrument. The neural components of consciousness divide into two distinct categories; the organizational state of the neural networks at any one-time and the processes or traffic of electrical signals across these networks. The former determines whether a person is conscious - awake, or unconscious and the latter accounts for the experiences of conscious awareness. Biological changes of depression destroy the harmony of these neural underpinnings.

\subsection{Neurochemistry}

With increasing knowledge of the physiological functions of the monoamine neuronal system, the monoamine hypothesis is still in the forefront of scientific research. The limbic system and hypothalamus are concerned with the control of emotion, motivation and memory. The actions of monoamine transmitters in the brain are related to emotional responses. Even though the neurotransmitter profile is incomplete, their distribution within the brain has been mapped. Though the pathophysiology of depression is still poorly understood, it is thought that abnormalities of noradrenalin and serotonin in localised areas of the brain that are largely concerned with mood and behaviour have some involvement in the genesis of depression at the physical level.

Chemical transmission is one of the methods by which neurons exchange or transfer information in the brain. We each have from 10 billion to 100 billion neurons within our brains. The chemical impulses or neurotransmitters, released by the axon, carry messages from one neuron to another. The chemical messenger passes between them through a narrow gap called a synapse that separates the neurons, and the message is picked up by the dendrite of the neighbouring neuron. The whole process takes only a few milliseconds and is the culmination of many steps [5]. The neurotransmitter is usually synthesised within the neuron and then transported and stored, prior to its release, at the presynaptic membrane. Once it is released to the synaptic cleft, the neurotransmitter binds to its specific receptors and its action is terminated by diffusion, metabolism or re-uptake into the presynaptic neuron. There are several lines of evidence for abnormal noradrenergic and serotonergic function in depressive disorders. It is unclear whether changes in levels of neurotransmitters are instrumental in the development of depression or depression causes the changes in neurotransmitters. Antidepressants effect various neurochemical changes; however, their specific antidepressant effect remains unknown - the biochemical changes have not been described by a single model or theory. It is now believed that many neurotransmitter systems, acting at various sites within the brain, participate with neuroendocrine mechanisms in the pathogenesis of depressive illness [6].

\subsection{Neuroendocrinology}

Endocrine and electrolyte imbalances are also biological correlates. The neuroendocrine axis (hypothalamo-pituitaryadrenaline axis) is often altered in depressive disorders and may be the mediator of environmental influences. Alternatively, it may be the primary cause of depression in certain depressive subtypes. It has been found that a great number of depressed people have abnormal levels of some hormones in their blood stream despite having healthy glands. In addition, those with endocrine problems such as Cushing's syndrome, Addison's disease and thyroid disorders are noticeably affected by depression. Hormonal levels in the blood are constantly monitored within the body. When a specific hormone rises to a particular level, normally the relevant gland stops production and halts release of the hormone. This feedback process is found to be impaired in those with clinical depression. Hormonal abnormalities are therefore intertwined with the altered brain chemistry recognised in depression. The hypothalamus regulates the pituitary gland, the conductor of the endocrine orchestra, and consequently controls the hormonal secretion of other glands; and the hypothalamus uses some of the neurotransmitters associated with depression. Blood levels of cortisol, which is secreted by the adrenal gland, are found to be high in nearly half of clinically depressed individuals. 
New techniques of fluorescent histochemistry, and immunohistochemical and in situ hybridisation, have permitted the elucidation of chemically defined neural circuits, providing tools for the burgeoning field of neurochemical pathology. Novel biological concepts of depression have recently emanated and they involve other receptor systems or intracellular targets. There is no doubt that depression may also stem from purely chemical causes. When depression is treated medically, there is great pressure on psychiatry to provide quantitative measurements, but such biological tests have detractors as well as advocates. Tests may reinforce the view that there is a dominant biological causation for all forms of depression. The author does not question the value of the biological information painstakingly gathered over the years but is dubious about the extreme biological view held by some medical scientists. A detailed discussion of the biological aspects of depression is beyond the scope of this paper.

\section{The Unconscious}

Unconscious factors have been always recognised as being implicated in the causation of depression, but with the popularity of reductionist views of mind, depression became misunderstood as a pure biological condition in the medical world. The present discussion would become fruitless without a clearer concept of the unconscious.

The concept of the unconscious mind - the source of dreams and automatic thoughts, the storehouse of forgotten memories, and the locus of implicit knowledge - existed, with different terminologies, throughout the centuries before the advent of Freud. The ultra-fast primary process thinking of the unconscious violates the laws of classical psychology. The study of quantum physics has advanced to such an extent that we are now in a position to achieve a deeper perception of the unconscious, without which we are unable to study the mechanism of its functioning and the causes of depression.

The prevailing reductionist model of mind limits the etiological study of depression. Quantum opens new windows of research opportunity. Quantum-cumhypnotherapeutic and cognitive views of depression may reveal some missing links that should be correlated with psychodynamic approaches. The hypnotherapeutic view that depression is a network of negative trance states of a neuroquantological nature has transpersonal psychiatric significance. It is arguable that, while hypnotherapy may not have any practical value in the amelioration of depression, it has a significant contribution to offer to its decoding.

For a deeper non-chemical understanding of depression, study of unconscious mental processing is vital. After a hundred years of controversy, consciousness remains the tip of the psychic iceberg that Freud promulgated, [7] and quantum physicists appear to support his metaphor. Freud meant only unconscious cerebration when he introduced the all-embracing term of the unconscious [8]. Unconscious events are not observable, and that is the fundamental obstacle to the integration of Freudian views of mind and the established sciences. Similarly, quantum wave function cannot be observed; it can only be inferred from observable data. In spite of that, quantum physics has become a recognised science. As it happens, the only way of integrating Freudian concepts with science is through the mediation of quantum physics.

The behavioural/cognitive outpourings of the second half of the $20^{\text {th }}$ century was an attempt to make psychology look more scientific. Consequently, consciousness became described as being awake and alert and the very term consciousness nearly disappeared from the map of cognitive sciences in the second of the $20^{\text {th }}$ century [9]. The behaviourists failed to distinguish the conscious and the unconscious mind. The novelty of quantum physics is that it can explain the distinctions of the two modes of consciousness. The unconscious is consciousness without awareness, when no quantum collapse takes place [10]. In other words, awareness arises with quantum collapse and the unconscious becomes operational when awareness is not, i.e. when there is no quantum collapse. The unconscious is the source of quantum thinking or associate thinking and there may be more than one mode of such co-thinking. We may also assume that more than one such non-classical thinking may be taking place parallel to the classical thinking. The creative quantum leaps involve quantum jumps from one such parallel thinking to another in a discontinuous manner and cure of diseases is also assumed to involve quantum leaps of creativity [10]. This phenomenon is analogous to the electrons circulating in the nuclear orbit disappearing and reappearing without using the intermediate space- the quantum jump. Both the conscious and unconscious mind may be powered by the quantum consciousness.

Amit Goswami has opined that humans suppress the memories of traumatic experiences so deep that consciousness rarely collapses them leaving them to unconscious processing [6]. These memories breed in the unconscious level and are processed by producing biological effects of disease. We become unaware of these memories because we never collapse these memories in our conscious thoughts. These suppressed memories create blocks in the flow of vital energy leading to malfunctioning organs and we experience them as diseases [11]. The experience of depression is no different from physical illness, except that it is experienced at a conscious level in cultures with advanced language where depression is accepted as a disease entity and their language is rich in words that would facilitate the free expression of depressive feelings. It is now well recognised that depression is somatised in cultures with poor linguistic facility for the expression of depressive thoughts (eg, Asian cultures), and even as psychosis in cultures where feelings of depression is stubbornly inhibited and internalised ( eg, African cultures).

\section{Quantum Based Cognitive Views}

The main stream of psychiatry holds the view that depressive thoughts are secondary to a primary disturbance of mood. Beck, by suggesting that depressive cognition may 
be the primary disorder, saved psychiatry from developing a pure biochemical model of depressive illness [12]. Mind processes meaning to life experiences, but if the mind is faulty, it is bound to generate faulty meanings. In depression, the mind becomes akin to a negatively programmed computer. The unconscious mind is the seat of parallel or quantum thinking and cognitive depression stems from deranged quantum thinking. Beck divided the depressive cognitions into three groups: 1) negative automatic thoughts, 2) a set of unrealistic expectations, and 3) a series of cognitive distortions. He further classified cognitive distortions as follows:

a) Arbitrary interference - drawing a conclusion when there is no evidence for it and even some against it;

b) Selective abstraction - focusing on a detail and ignoring more important features of a situation;

c) Overgeneralisation - drawing a general conclusion on the basis of a single incident;

d) Minimisation and magnification - performance is underestimated and errors are overestimated; e) personalisation - referring to patients' proclivity to relate external events to themselves when there is no basis for making such a connection;

f) Absolutistic - dichotomous thinking is manifested in the tendency to place all experiences in one of two opposite categories; for example, flawless or defective, immaculate or filthy, saint or sinner. In describing himself, the patient selects the extreme negative categorisation.

These depressogenic mechanisms are thought to be present in patients long before the onset of depressive illness. According to the cognitive model of depression, depressive symptoms feed each other and do not produce depression, but they generate and perpetuate NBD. These cognitive distortions are so systematised that one cannot explain them in terms of aberrations of neurotransmitter transmission and cannot be following the classical logic of conscious mind. How could it be if neurotransmitters are dysfunctional, such organised thinking is possible? These depressive cognitions could be better explained in terms of quantum misrepresentation and they are derivatives of deranged associate thinking.

\section{Hypnotherapeutic Views and the Quantum Links}

\subsection{Quantum Hypnotherapeutic Views}

Hypnosis may be defined as a state of intensified attention and receptiveness and an increased responsiveness to an idea or a set of ideas [13]. This is akin to a quantum state of consciousness relating quantum entanglements. The power of hypnotism can be easily accommodated in a science within consciousness [11]. Goswami argues that the ego individuality is unreal and it is an identity consciousness puts on; consciousness has the ability to alter this identity to shirk the specific ordinary ego demands of the physical world upon us. Classical world is inflexible whereas quantum world is expandable and bizarre. Hypnosis involves a spectrum of consciousness. It offers a way to conceptualise how human beings construct their individual realities, and how they interact more effectively with others through quantum entanglement.

Hypnotherapy is a meeting point for biological and nonbiological aspects of depression, and also an interface between quantum and brain. Hypnotherapists have always been opposed to the tradition of labelling depression without any identifiable psychogenic stressors as 'endogenous or biological depression', and they subsume all the diagnostic categories under the broad term 'depressions'. Like psychoanalysis, hypnosis is a powerful quantum research tool, but it has questionable therapeutic value in treating depression [14]. It is effective in accessing the subconscious, which may be a non-ordinary state of consciousness. The experience of an altered state of consciousness is part of our human psycho-biological heritage. If depression is a state of symptomatic trance, everyone is prone to it, and like any other quantum trance state it may be interrupted. Hypnotherapists claim that the human psyche is more amenable to positive changes when in the hypnotic state than in the ordinary thinking mode. Little attention has been paid to understanding depression from a quantum and hypnotherapeutic viewpoint.

Under hypnosis, a person experiences time distortion. Space is also distorted, and the body may have sensations of heaviness, expansion, lightness, flotation and elevation. These experiences tally with quantum physics and are representative of the intersection between hypnosis and quantum physics. Time is a property of the conscious mind whereas the unconscious mind perceives time and space as one, time being a dimension of space. Time is linear in the conscious mind, and it is non-linear in the unconscious. The conscious mind may be regarded as functioning like a neurocomputer and one of the different strata of the unconscious may be likened to a quantum computer. The memories collected in the conscious mind are limited and distorted owing to space-time separation, whereas the memories imprinted in the unconscious are the original ones and together they represent one's entire life history [15]. Hypnotherapy is reliant on the flexibility of the quantum unconscious mind whereas the material brain-related classical mind is inflexible like the classical natural world. The quantum unconscious being highly flexible like the quantum world, hypnotherapists claim to be able to manipulate and remould it to suit the inflexible realties of the classical world that the patient has to face. The popular saying that it is all in the mind should be modified as, 'It is all in the quantum mind.'

\subsection{Depressive Quantum Trance States}

Kräupl Taylor suggests that the psychogenesis of posthypnotic symptom formation has offered a prototype for theories of dynamic psychopathology that is helpful in studying and explaining how neurotic symptoms germinate [16]. In a similar fashion, various trance phenomena may 
shed light on our understanding of the formation of different forms of depressive experience. Hypnosis amplifies subjective experiences in a positive or negative way. In depression, it amplifies feelings of anger, guilt, and hopelessness and so on, even though the patient does not deliberately seek these. The development of 'cognitive depression' is a slow process, the product of self-induced trance states of varying intensity, occurring either continuously or periodically. We do not know how it is initiated without any conscious effort on the part of the patient. According to the school of hypnotherapy, depression is a lifestyle permeating all dimensions of human experience.

In the negative trance state of depression, every new stimulus is put through a negative filter and the depressed patient's realities become distorted. Just as a patient with thalamic syndrome perceives every stimulus as pain, in the negative trance state the individual takes in all new stimuli as psychic pain. Age regression, age progression, amnesia, analgesia, anaesthesia, catalepsy, dissociation, hallucination, ideodynamic responses, sensory alterations and time distortion are the building blocks of all subjective experiences. Michael Yapko suggests that depression pictures most of these classical hypnotic states and he thus identifies eight such trance states responsible for the spiralling of depression [17]. The negative trance states of depression follow the disturbed quantum logic of the unconscious and would appear bizarre for the conscious mind.

Depressed patients suffer from the tyranny of memories and fight against the dinosaurs of the past. Their dwelling in the past is similar to the trance experience of age regression. Likewise, patients who are focusing on the future with a pessimistic attitude are inducing negative age progression unwittingly. They project past hurts into the future and become hopeless. Consequently, they cannot see 'any light at the end of the tunnel'. Beck and his colleagues consider hopelessness to be the driving force in suicide [18]. Hopelessness is the outcome of negative age progression. People who commit suicide go into a trance state, convincing themselves that suicide is the most rational choice that is left to them. Because of the continuous flow of negative thoughts, positives are forgotten by them. Yapko argues that patients who are unable to recall their past merits and achievements consciously are in an amnesiac mental state [17]. The inhibition of voluntary movement is defined as catalepsy, and hypnotically induced catalepsy is very much like the cataleptic state of depressed patients who are deeply absorbed in their sad musings. Such patients exhibit psychomotor retardation and rigid posturing.

The trance phenomenon of dissociation is very prominent in depression. Firstly, there is dissociation between internal experience and the immediate environment. Secondly, the patient dissociates between negatives and positives. The barren negatives dominate their consciousness and positives are overlooked. Thirdly, there is a temporary dissociation when they are immersed in past traumas while withdrawing from the immediate demands of their current existence. So also, when people constantly evaluate themselves negatively, the parts of the self are dissociated. When depressive thoughts develop into a kind of automatic pilot that pushes patients to commit suicide, we have an example of the quantum mind taking over the brain functions in entirety. The quantum unconscious mind dissociates itself from the classical logic of ordinary consciousness and follows its own logic. Depressive thoughts develop into a 'quantum automatic pilot' and push the patient to commit suicide.

Yapko contends that the automatic thoughts and automatic feelings typical of depressive patients are to be considered as ideodynanic responses of the trance phenomenon [17]. By definition, if positive hallucination is perception in the absence of sensory stimuli, negative hallucination is absence of perception in the presence of sensory stimuli. Depressed patients perceive rejection and negativity when they do not exist in reality and exist in a quantum mode. Similarly, their inability to appreciate positive feedback is comparable to negative hallucinatory experience.

In hypnosis, sensory experiences of any modality may be diminished or enhanced. Somatisation of the depressed patients is a sensory alteration. Sensory capabilities are generally reduced in depressed patients. As a result, sensory experiences become dull, leading to loss of libido, to food becoming tasteless and to general anhedonia. For them, days appear darker than nights. Their internal clocks are set at a slower rate to those of others. Time distortion is evident when patients say that a day is like a month. Because of time distortion they cannot see an end to the unhappy events in their lives. These patients' metronomes are tuned at a slower rate than normal. According to Yapko, the extended experience of past and current unhappiness and the shortened experience of future possibilities are also a manifestation of time distortion [17]. Clance recognises that a trance state is involved in the impostor phenomenon [19]. Capgras syndrome is the popular psychiatric term for the subjective double experience, and it is also observed in severe depressive disorder; a quantum distortion of the unconscious mind.

The concepts of repression and dissociation traceable to the works of Freud and Janet have dominated the practice of modern psychotherapy. That process is thought to aid the recovery of painful feelings and memories buried in the unconscious mind. The theory is that unearthing the memories leads to clinical improvement, but a recent survey of experimental studies of memories challenges the dependability of this conclusion and draws attention to its shortcomings. Experimental studies involving the use of hypnosis demonstrate that inaccurate memories or confrontations are at least as common as accurate retrieval in hypnosis [20]. The patients are already haunted by the past, and that in itself is a compelling reason for caution in using age regression for therapeutic purposes in depression. The discussions in the preceding paragraphs demonstrate that trance states involved in depression do not follow classical logic, but a deviated logic; a form of the quantum logic of the unconscious mind. Quoting from the observations of Tarlaci [21], after all, among other commonalities, it is also the 
bizarre nature and incomprehensibility of consciousness and particle physics that attract these concepts towards each other.

Unlike psychoanalysis, quantum hypnotherapy focuses on the structure and process of thoughts rather than the content. A recent meta-analysis has established that hypnosis enhances the efficacy of both psychodynamic and cognitive behavioural therapies [22]. But hypnosis is not a panacea or a substitute for physical and psychotherapeutic treatment methods. Irvin Kirsch considers hypnotherapy an empirically validated, non-deceptive placebo, the effects of which are mediated by response expectancies [23].

Self-hypnosis is to be discouraged until cognitive reprogramming is achieved as it may reinforce existing cognitive disturbances that occur in depression. Hypnosis is contra-indicated in actively suicidal patients and patients with psychotic symptoms. Experts in the field of hypnosis have either totally ignored or openly suggested that hypnosis is contra-indicated in the treatment of depression [24]. The traditional view regarding the use of hypnotherapy for depressed patients is that extreme caution needs to be exercised. Its unitary use is not favoured. The hypnotherapeutic views of depression are complementary to the ethico-bio-psychofamilio-sociocultural model proposed by Vernon Neppe [25].

\section{Non-biological Factors}

Many depressed patients are apparently trapped in a network of human manipulation, and that they themselves are basically, well-integrated individuals. Their depression may be the outcome of a subtle and highly manipulative network whose perpetrators may be even in the spiritual realm. NBD can thus be the result of a subtle and highly controlling network phenomenon whose executors can be in the spiritual realm as well [26-32]. The sociological influences on these patients are intertwined with psychological and $p s i /$ spiritual factors and the result is depression. Sociological factors also imply extrinsic $\mathrm{psi} / \mathrm{spiritual}$ influence from fellow humans. Further, in this age of globalism achieved through the influence of technology, there is a worldwide responsibility as well.

Depression has its psychological, biological, sociological and $p s i /$ spiritual aspects, and the biological aspects of this multidimensional phenomenon should not be underestimated. Through spiritual influence, human behaviour also has a para-psychodynamic dimension. The prevailing reductionist model of mind is not at all helpful in appreciating the nonchemical factors contributing to the genesis of depression.

Brain chemistry may be influenced by internal psychokinesis and also by extrinsic psi factors. The spiritistic realm is an entity that contains discarnate spirits as well as unknown spiritistic forces. These spirits may be an element within the spectrum of circumstances that together constitute depression. Just as living beings contribute to the causation of depression, the interaction of the human mind and the nonphysical world may contribute too. To study and cure depression, it is vital to recognize that humans are not electrical animals but are endowed with an aesthetic dimension. The succeeding paragraphs are therefore concerned with presenting a challenge to the models of mind based on Newtonian and Darwinian sciences.

\section{Mind, Consciousness and Para-psychodynamics}

\subsection{The Imperceptibility Problem}

One of the impediments of appreciating an invisible realm is the Newtonian based world view promulgating that nothing exists beyond the objective world. In such a scenario, non-biological factors of depression become less relevant and even unconceivable. There are many fences that have to be jumped off to appreciate the non-biological causation including psycho-spiritual factors of depression. The view held by classical physicists that two objects cannot occupy the same space at the same time has been the scientific block to accepting the existence of an unphysical/non-physical component in association with the brain. A discourse of this cardinal problem is germane to understanding depression from a quantum perspective. The same confusion is restricting scientists when considering a para-psychodynamic interpretation of depression. Robert D. Klauber states that two subatomic particles can exist together, just as two waves rolling over the ocean and heading in opposite directions pass through each other unhindered, occupying the same area of the water's surface for a time [33].

The discovery of neutrinos has been a breakthrough in the thinking of post-Einsteinian scientists and Klauber found a solution to the imperceptibility problem in neutrino physics [33]. These are tiny, subatomic particles without any electrical charges, and because they do not couple with electromagnetic forces their presence goes undetected. Most neutrinos pass through the earth unscathed. We perceive them only when particles in our sense organs are coupled to the particles transmitting particular properties from the object; if there is no coupling, there is no perception. Trillions of neutrinos pass through matter without our being aware of their presence. Klauber submits that we may imagine the existence of similar unknown particles that do not couple with the physical fields constituting other worlds which coexist with ours [33]. Only left-handed neutrinos (spinning in a counter clockwise direction) could interact through weak nuclear force with another particle and have been detected; right-handed neutrinos (spinning in a clockwise direction) have escaped detection, though they may well exist - righthanded electrons and quarks have been proved to exist. There may be a variety of neutrino-like particles, all tenuous and imperceptible, and it may be conjectured that the extracerebral component of the 'mind stuff' may be partly or fully composed of such diverse particles, but our body is made up of leptons and quarks. The former is unphysical and unobservable by present-day instrumentation. 
Subtle realms may be made of 'neutrino-like particle families' that coexist with our physical world without our being aware of their existence. Thus, extra dimensions have been postulated, based on the evidence of elementary particle physics, and the possibility of our consciousness drifting over to other dimensions has been explored [34, 35. 36, 37, 38]. In other words, humans and all the particles in the standard model are trapped in the three dimensions of a higher dimensional world and right-handed neutrinos may be free to roam around the multidimensional universe, escaping detection.

\subsection{Brain-Mind-Consciousness Complex}

Mind and consciousness are distinguished as separate in Eastern Philosophy whereas they are treated as one entity in western thinking. The concept of mental space is helpful in understanding some subjective experiences. The physical body is in physical space, and the minds of humans exist in a personal mental space - a higher dimensional space existing like a satellite spatial system that is occupied by minds, or at least by components of minds [39]. This concept is supported by particle physics. Mental events occur in a space that is different from the space we occupy in everyday lives as well as from the physical space that Classical physicists describe. The mental space is an entirely personal one and is protected from the intrusions of other person's thoughts. Dr Ian Stevenson, who is a supporter of this hypothesis, states that the barriers of the mental space weaken on rare occasions, when we experience unusual telepathic and paranormal communications [40].

Mind according to Stevenson is that private part of the person where only he can get access through the process of introspection [40]. It is a brain-mind-consciousness complex. Mind may be a folder of consciousness, a neural ending of consciousness. Psyche and soma are recognised as correlated possibilities of consciousness and changes in the physiological states would affect the mental state and vice versa-somato psychic and psycho-somatic interactions. The greatest hindrance to appreciating the psychosomatic concept has been the Cartesian dualism that prevailed over centuries. Mind and consciousness were considered identical in Cartesian philosophy. Particle physicists tend to distinguish between mind and consciousness, but they are interdependent. Once the primacy of consciousness is accepted, there is no need for dualism; consciousness acts as a mediator between mind and brain. Mind is not functioning like a computer because computers cannot process meaning.

The mind and the brain coexist in the same way that a coloured aqueous solution and a sponge coexist in the same locality. The colouring pigment represents the spiritual factor of the mind. Stevenson introduced the analogy of a motor vehicle to explain the functional dualism existing in the mind-brain union. A motor vehicle may be functionally divided into two by pressing the clutch [40].

In the quantum mechanics of consciousness, there are three views prevailing. The first is the reductionist understanding that that brain evolves quantum consciousness.
The second is that brain seizes consciousness from somewhere in the universe and that proto-consciousness exists - property dualism. The third opinion propagates the pre-existence of consciousness even before the Bing Bang and that consciousness is the fundamental driving forcesubstance dualism. All the three views together could lead to the formation of an integrated theory of consciousness.

\subsection{Biophysicist and Spiritual - Triune Model}

C. D. Broad put forward a compound theory of mind [41], suggesting that mind, as we know it, may be a compound of two factors, neither of which separately has the properties of mind. One of these is the bodily or organic factor, the other a psychic factor or psi component. The psychic component, he suggests, may persist after death. To say merely that the psi component will survive after death is a very loose assertion. Robert Crookall's speculations about mind are very close to, but more specific than, those of Professor Broad. Based on Crookall's observations, we may modify Broad's compound theory of mind. Without introducing any extravagant supposition, such a modified theory is sufficient to explain discarnate survival [42]. The psi component hypothesised by Broad corresponds to the psychical body of Crookall and consists of components from the soul body and the spiritual body. We may refer to the spiritual body as the nonbiological body. The spiritual body - which may be conjectured to be composed of micro-micro-micro particles survives after the extinction of the ordinary human body. The super psi may be a property of the spiritual body and probably is indirect evidence of its existence.

It is the evidence for post-mortem existence that makes the hypothesis of spiritual body attractive. It would perhaps be desirable to stick with an established word, 'spiritual', no matter how inappropriate it may be in scientific terms, rather than to create more confusion in this already very loaded subject by introducing more terminology than is necessary. As we have seen earlier, the spiritual body is the subtlest and the most pervasive of the three components and a nonphysical continuum is maintained between the three by the all-pervasive non-biological component.

Myer's concept of subliminal self [43] is different from the Freudian concept of the unconscious. His subliminal realm consists of a hierarchical organisation, each with its own functional properties. Myers tried to describe three such levels. The lowest stratum is that of bare vegetative function. The middle realm is the hypnotic stratum and is associated with automatism and deep hypnosis and is the source of dreams and imagination. The third and deepest realm is the most mysterious and is the locus of psi phenomena and the intuitions and inspirations of creative people. Interestingly, Myers' model of the subliminal realm is in tandem with the plasma physicists' concept of different energy bodies assembled like an onion ring and constituting the human body and psyche [44]. Freud's unconscious mind was vague and good enough only to explain neurotic disorders, but not useful in elucidating psychotic symptoms. Quantum based unconscious mind is more illuminative in this line of research. 
According to certain schools of Quantum physics, consciousness is the ground of matter and mind, and they are both the possibilities of consciousness. The quantum collapse resulting in the actuality of matter and mind is the causal power of consciousness and it is downward causation as opposed to upward causation which consists of elementary particles making conglomerations of atoms, atoms making molecules, molecules forming the cells, the body including brain and the brain that makes consciousness and mind [45]. Goswami also proposes that with quantum collapse, consciousness contains five compartments of possibilities and they are the material body, the vital body, the mind, the supramental intellect and the blissful body [46].

Mysticism is the bedrock of reality [3] and so humans may be endowed with a mystical consciousness. Bernard Haisch proposes a consciousness that may be a type of ultimate consciousness [47, 48]. It might have evolved in a higher dimensional realm and was effectively downloaded into the quantum computer that is shaped from proto-consciousness [49]. Charles Tart's conjecture of a possible non-objective centre of personal identity and consciousness may match with the ultimate consciousness [50]. Stokes has termed multiple spheres of consciousness within each person 'miniShins'. He has constructed an adaptation of the term 'Shin', originally devised to refer to the soul [51]. According to Stokes, each mini-Shin co-existing with the brain may receive input from a widespread area of the brain and may have its own information-processing capacity. Mini-Shins may have only limited executive powers. Stokes's model is distantly similar to Daniel Dannet's multiple drafts model of consciousness without a 'Cartesian theatre' and excluding the intractable problems [52]. But it may be postulated that in supervision of mini-Shins there may be a vestige of potential consciousness -an over-Shin or super-Shin which may be the substratum of a higher consciousness.

Without extending the boundaries of scientific arena, one may also postulate that a higher consciousness (quantum-like consciousness or spiritual consciousness) may have its foundation beyond the space where dreams occur as dreams and consensus realities may be generated by an identical mechanism-quantum consciousness. Deepak Chopra seems to identify quantum consciousness as an interface between brain and spiritual consciousness [53]. Belief in the reality of dreams is based on inter-subjective agreement rather than on full scientific evidence. Likewise, the existence of a greater mind or spiritual consciousness is based on inter-subjective agreement because humans know intuitively that they are autonomous beings and have their own first-hand human experiences. The Spiritual consciousness is singular and all pervasive. Thus, we have a biological self and a spiritual self.

Reductionists proclaim that consciousness is like the rainbow over the waterfall and likewise is an epiphenomenon of brain activity. Rainbow is not just water droplets; it becomes rainbow only when the sunlight reflects over the water droplets. Reductionists are unable to explain where the sunlight comes from. The reductionist analogy can be therefore twisted to present a non-reductionist analogy- the celestial body is comparable to the sun reflecting on the water droplets and the brain activities merely form a substratum for the spiritual body to manifest. 'By reducing the secret of the rainbow to the colours of a prism [22],' we may be inching close to appreciating our ultimate consciousness.

Consciousness is being identified as a fifth force; gravitational force, electromagnetism, weak and strong force being the other four forces. Because consciousness is dimensionless, it is inappropriate to use the term 'force' and therefore, consciousness may be designated as the 'Fifth Influence [54].' But, consciousness may have an objectivity in its own right. The biophysicist and spiritual tripartite view of the mind accommodates all forms of extra-sensory phenomena. The above parallel does not in any way diminish the immensity of the dichotomy between matter and spirit, but simply illustrates that they are accessible to one other. Considerably more work needs to be done along these lines before any of these conjectures can be defined convincingly.

\subsection{Psycho-Spiritual Causation}

NBD may be viewed differently once the triune model of brain-mind-consciousness complex is accepted. If the existence of spiritual dimensions is accepted, the influences of discarnate spirits and other external intellectual entities become another facet of the social aspects of human life and depression becomes a psycho-bio-social and spiritual condition. Human behaviour may have parapsychodynamics. Newtonian views of denial of the invisible realm reached its zenith in the second half of $20^{\text {th }}$ century in cognitive sciences and the consequence was over medicalisation of depression. Depression has to be neutralised by positive life events, but such life events are not easily available, but positive spiritual strokes are readily available-happiness is the reflection of what we do for others. The belief that a heavenly dimension exists to assist the humans in their earthly plights can be a comforting thought when the patient is helpless and hopeless-there are more helpful angels than stars in the sky!

The idea of spirt attachment has been extensively written about. It is not the purpose of sincere spirit releasement techniques to place all the blame and responsibility on outer sources, but it is the individual's own thoughts and emotions that open the person to any outside stimuli. Responsible therapists make cautious warning to their satisfied clients that unless the individual invests some conscious effort in maintaining healthy mental state and body, he may very well open himself to more unwholesome inspirations.

Personality disorders and sudden onset of deviant behaviour could be triggered by psycho-spiritual factors and may have abnormal para-psychodynamic causation. One of the world authorities on mediumship literature, Michael Tymn writes [55], "Authorities involved with the investigation of the Las Vegas shooting massacre and the more recent Austin bombings are mystified as to what motivated such deviant, insane behaviour. Neither the shooter nor the bomber seems to have had anything in his 
past to suggest he was capable of such a horrendous act. There is, however, a possible explanation that no authority dares mention, as the person would be ignorantly laughed if he or she did. I'm referring to possession, or even lesser influence by devious 'earthbound spirits.'

\subsection{Consciousness Based Medicine}

With the progress in consciousness studies, the view that depression is a disorder of consciousness is getting popular and consciousness-based medicine has started emerging in different quarters of healing professions. Spiritual therapies are also gaining ground as a last resort for therapy resistant depression. Change of life style and a philosophical outlook towards life are the basic principles of such therapies. The U. S. anaesthetist, Dr Rajive Parti who had a near death experience (NDE) is a strong advocate of such a healing medicine [56]. Parti has a downward approach to depression as opposed to the upward approach of reductionist scientists. There are approximately 10000 documented cases of NDEs challenging the reductionist model of consciousness. The subjective experience of depression may respond to consciousness-based healing methods, but the tormenting psychic pain has to be addressed through the highly precious psychotropic medications.

The consciousness-based medicine Parti promotes is based on several eternal truths [56] such as: a. Consciousness exists outside of the body. b. There is life after death. c. In isolated cases, certain people may have past lives and their experiences therein can shape their current realities. d. We are all connected to each other because we are all made of the one and same energy that manifests as differentiated matter. e. Divine beings exist to help and guide us. f. There are different levels of consciousness. g. There is one, allpervading, supreme love and intelligence that is the source of the entire universe, and that love is the supreme source of creation. In this juncture, the late Dr Kenneth MaCall's insightful contributions to spiritual medicine are to be recognized [57, 58, 59]. Similarly, Deepak Chopra has linked the prescientific Ayurvedic wisdom with physics and neurosciences. He has found a "network of intelligence" in the human body with the potential of healing bodily and psychological disorders [60].

\section{Clinical Neuroquantology}

There is a paucity of literature about the practical value of parapsychology in clinical practice. Even though Jan Ehrenwald documented cases indicating that the psi hypothesis can assist in the understanding of psychiatric disorders [61-64] - particularly schizophrenia - he did not elaborate on the contribution of parapsychology to the determination of the causes and cure of depression. The quantum elements of depression described in this paper justifies further exploration. Neuroquantoly has impressively offered explanation for the puzzling secondary symptoms of schizophrenia from a quantum perspective [65-68]. Using catch phrases from quantum physics, it may be asserted that most cases of depression may be due to 'downward causation' and schizophrenia and bipolar disorders may be due to 'upward causation.' Even though bipolar disorder is essentially a biological disorder, it is now assumed that the manic experience may have some contributions from the quantum part of the mind if Penrose-Hameroff hypothesis of Orchestrated Objective Reduction of Quantum Coherence in Brain Microtubules is valid [69, 70], an instance how consciousness colour psychiatric symptomatology.

The conscious mind follows classical logic and the unconscious mind follows quantum logic and operates much faster. Paola Zizzi and Messimo Pregnolato notice [68], 'For a healthy mind the passage from the unconscious state to the conscious state is marked, according to the Orch-Or model of Penrose and Hameroff, by adecoherence of tubulin qubits. This may have understood in terms of very fast switches from the quantum logic of the unconscious to the classical logic of consciousness.' They also argue that in schizophrenia these switches are not fast enough, and therefore, the schizophrenic mind remains trapped in the unconscious logical mode too long. One may hypothesise a 'quantum gate' built of microtubules controlling and filtering the passage of energy and information parcels from the quantum mind into the material brain that might help to explain some of the psychopathological experiences involving the both modes of consciousness. This aperture is similar to the 'doors of perception' conjectured by Aldous Huxley.

Normally the quantum gate is able to tune with the ultrafast oscillation of consciousness between the quantum mode of the unconscious to the classical mode of the conscious mind. An insightful schizophrenia sufferer stated, 'Healthy brains are able to block my realities which are very real to me,' and another patient commented, 'Everybody's reality is in my background and my extra reality is in their background.' These statements would make sense only when a neuro-quantological approach is applied to the primary process thinking of schizophrenia.

In bipolar disorder the operations of the quantum gate may be less efficient or deranged. In such a scenario, the cognitive emptiness and emotional flatness of bipolar depression could be explained as due to closure of the quantum gate and consequent blocking of the energy and information parcels from the unconscious mind. The hypomanic/manic racing of thoughts and excess energy levels may be due to the uncontrolled opening of the hypothetical quantum gate allowing excess transfer of energy and information parcels from the quantum mind.

Allopathic medicine is not a remedy for all the problems of mind and body and likewise, alternative medicine practices are also constrained, but if they could be discerned and applied appropriately, many patients would benefit immensely. Amit Goswami offers a very plausible theoretical explanation as to why they both work within the unifying framework of Quantum Physics. He has also written amply about why and where they should be used in treating the body and mind. Goswami's insightful renderings are a valuable contribution to clinical neuroQuantology [11]. 
It appears that NeuroQuantology is coming of age, and if one combines the wisdom gathered through parapsychology, hypnotherapy and NeuroQuantology, it is appropriate to establish even a subspecialty of Clinical NeuroQuantology. Hypnotherapy could grow as an effective research tool under the auspices of clinical NeuroQuantology. Consciousness studies have great potential significance in relation to psychiatric disorders from both etiological and therapeutic points of view. In their determination to squeeze consciousness into a tight neurobiological model, neuroscientists are missing a precious opportunity to participate in the emergence of a new paradigm -the search for the causes and remedies for psychiatric disorders within the frame work of clinical NeuroQuantology. It is now well recognized that a quantum mechanical body and brain may be co-existing with the material body and that may be unphysical only with the present-day instruments [71, 72]. Hopefully, Clinical NeuroQuantology would accelerate the evolution of psychiatry ultimately as 'Enlightened Psychiatry.'

\section{Antidepressant Therapy}

Antidepressants are a highly precious contribution to the psychiatric armamentarium from psychopharmacology and they are safe in the hands of clinicians who are cognizant of the non-chemical factors of clinical depression. Conventional antidepressants are being superseded by modern medication. The potential of non-monoaminergic antidepressants - which are a branch of the current monoamine-cycle-based antidepressants - is being explored. In recent years many new potentially relevant brain transmitters and proteins have been identified. A number of neuroleptic-based approaches are being made to develop novel antidepressants.

Antidepressants have not shown convincingly any superior effect than placebo or to affect depression and suicide rates and so their uses warrant further review is an interesting finding [73]. The word antidepressant itself may be a misnomer. They are probably only anti stress agents or mood lifting agents. But the wording has given the wrong message to patients as well as to mental health professionals that these drugs would cure depression. Consequently, psychological aspects of depression are most often over looked; depression becomes perpetuated [74]. Antidepressants have a role in the treatment of depression but their role is not well defined. To use a medical analogy, anti-pyrexial agents have a place in the symptomatic relief of pneumonia but they are not to be equated to the antibiotic treatment. Likewise, antidepressants are helpful in treating the neurotransmitter problem of depression and they lift the mood, enhancing one's own mental resources to fight against depression. One cannot trivialise the neurotransmitter derangement; depression is as serious as cancer when patients are actively suicidal.

Between 20 to $40 \%$ depressed patients respond only minimally to mono-therapy [75]. Only $50 \%$ of patients not responding to a primary antidepressant improve when another antidepressant is introduced [76]. In other words, a clinician has the formidable task of looking after a good number of treatment resistant depressed patients. This situation has caused an alarming economic disability with serious implications in the bio-psychosocial spectrum and also therapeutic pessimism. The validity of the terminology 'treatment resistant depression (TRD)' and the new term "multiple therapy-resistant major depressive disorder (MTRMDD) [77]" is being questioned [78]. The truth may be that clinicians misconstrue these cases of NBD as instances of biological resistance.

Of note, it is now increasingly being recognised that many young patients suffering from persistent depression are actually suffering from premorbid bipolar depression or depression with bipolarity [79]. A subset of patients diagnosed with unipolar depression in fact suffers from bipolar depression [80]. Only when they declare hypomania or mania, they get diagnosed as bipolar disorder. In fact, antidepressants have questionable value in treating such cases. As a matter of fact, antidepressants could destabilise them and even lead to affective switch. When young adults do not respond to short courses of antidepressants, bipolarity should be ruled out. Bipolarity in disguise accounts for many young suicides.

Modern antidepressants are replacing the conventional ones. Branching out of the current monoamine cycle-based antidepressants, the possibility of non-monoaminergic antidepressants are being explored. During recent years many new potentially relevant brain transmitters and proteins have been identified. There are a number of neuroleptic based approaches to develop novel antidepressants. Various recent findings show that there are changes in neurotrophines or corticotrophin releasing hormones associated with depression. New interventions to stop the stress hormone cascade before it gets rolling are being thought about: an input to psychopharmacology from endocrinology. The CRF (corticotrophin releasing factor) blockers obstruct release of stress hormones that may contribute to depression, creating a cushion or buffer in the brain interrupting the biochemical signals of stress response. But these drugs in the pipeline are also running the risk of getting baptised as antidepressants.

Medical scientists are blindly concentrating on new chemical entities while innovating research and development aimed at producing very expensive and colourful pills to combat depression. The government, pharmaceutical industries and the medical profession, through the acceptance of only organic, genetic and biological aetiology have adopted a form of tunnel vision. Parallel to the introduction of new antidepressants, alternative medicine has been flourishing. Many of its approaches are based on the principles of progressive muscular and mental relaxation, and different schools of philosophy. Arnold Mindell [81] has opined that constantly going against pain and marginalising it is counterproductive and makes it worse, on the other hand, interacting with the pain by discovering the subtle sentient essence behind the experience would eventually diminish the pain or make it bearable; psychic pain is no different. Illness can be the best teacher. If depression is a consciousness 
generated illusion, consciousness must be able to cure it by streamlining the underlying cognitive belief system. The conventional medicine is based on material realism and needs integration with other forms of treatment methods that are less expensive. Biological psychiatry is comparable to classical physics in the sense that it is useful in the realm it is applicable. The growing popularity of alternative medicine among depressed patients reflects the failure and patients' frustrations from antidepressant therapy. Whatever theories of psychotherapies or drug therapy one may espouse, 'Human beings are the most important therapeutic agent for human beings.' After all, 4o\% of all drug effects including antidepressants are placebo effects and the quantum mechanical body may account for it [82]. Recently, there has been more uproar about the abuse of antidepressant drugs highlighting that they are only marginally more effective than placebo, but significantly more harmful than other forms of therapies [83].

\section{Conclusion}

Psychoanalysts have concentrated on eliciting past traumatic memories in order to identify the cause of depression in individual patients. The shortcoming of this initiative is that memories that are recalled over and over again may become inappropriately intensified and may acquire a significance that they do not merit. Patients undoubtedly benefit by opening what we may term the jewel box of the past, but not by unearthing the tin of worms of their infancy. Cognitive theories and Yapko's symptomatic trance views are complementary and fill some of the gaps in the research into depressive disorders. Beck's cognitive distortions are understood better in terms of quantum views of the unconscious than by being attributed to neurotransmitter derangement. Depressed people are in a negative quantum mode at a lower level of the brain-mind consciousness complex. More studies from a hypnotic and quantum point of view would help us to comprehend depressive experience as a network of different quantum trance states. Even sceptics regarding hypnotherapy have to acknowledge the value of such research. The biological aspects of this universal phenomenon should not be underestimated. The changes in brain chemistry and the biological symptoms in depression may be due to the wearing effect of long-standing stress on neurochemistry.

Beck has rightly stated that depression is the result of a cognitive revolution following a coup in the mind. That coup takes place in the inaccessible dark valleys of the unconscious mind, resulting in the formation of a negative hypnotic script. Cognitive theories cannot determine what initiates this development in the mind, nor can they explain the mechanism of how the unconscious mind prepares the negative hypnotic script. Does the process have an origin independent of the brain, one that in some instances stems from external intelligences? Cognitive therapy has to be amended to accommodate quantum views of depression. The quantum mind encompasses the gear box of different trance states.
Most cases of depression are considered to be psychobiosocial conditions, but there is a rare type of non-biological depression that it is hard to treat. The as-yet unidentified causes for this may include factors emanating from spiritual dimensions. These intractable occurrences may be the driving force for paradigmatic shifts in psychiatry. Thus, depression may be a psycho-bio-quantum and spiritual condition. The reductionist model of mind has been successfully challenged in recent times, and it is time for us to revise our concepts of depression in the light of such developments and the validity of consciousness-based medicine needs to be empirically proven. When patients suffer psychic pain, what they need urgently is relief from that pain, and that can be achieved only through using psychotropic drugs. Automatic thoughts may have their source in parallel quantum thinking. The avalanche of negative automatic thoughts beginning on the summit of the unconscious mind can be halted by a combination of cognitive therapy and medication.

Declaration of interest-none.

\section{References}

[1] Malik A Monsoor, James Lindesay. Quantum physics relevant to psychiatry. Neuroquantology 2009; Vol 7 (2): 314-317.

[2] Pylkkanen P. Can quantum analogies help us to understand the process of thought? Chapter 10. In Ed Globus GG, Pribram K. Vitiello G. Brain and Being. At the boundary between science, philosophy, language and arts. 2004.

[3] Coechi Messimo, Gabriell Fabio, Tonnele Lucio, Pregnolato Messimo. The Interactome Hypothesis of Depression. The Journal of Neuroquantology, 2010; 8(4):318.

[4] Chopra Deepak. Foreword to the Quantum Doctor Charlottesville: Hampton Roads Publishing, 2011.

[5] Stein J. F. (1982) an Introduction to Neurophysiology. Oxford: Blackwell scientific.

[6] Taylor MA, Berenbaum SA, Jampala VC. Are schizophrenia and affective disorders related? Preliminary data from a family study. American Journal of Psychiatry 1993:41: 949-58.

[7] Westen Drew. The Scientific Status of Unconscious Process: Is Freud Really Dead? Journal of the American Psychoanalytical Association 1999; 47(4):1061-1106.

[8] Kelly Edward; Kelly Emily Williams; Crabtree Adam; Gauld Alan; Grosso Michael; Greyson Bruce. The Irreducible Mind. Lanham: Rowman\& Littlefield Inc, 2006.

[9] Fenwick Peter. The Art of Dying. London: Continuum, 2008.

[10] Goswami Amit. Quantum Creativity. Cresskill, NJ: Hampton Press, 1999.

[11] Goswami Amit. The Quantum Doctor. Charlottesville: Hampton Roads Publishing, 2011.

[12] Beck, A. T. Depression; Clinical Experimental and Theoretical Aspects. New York: Harper and Row, 1967.

[13] Weitzenhoffer, A. M. The Practice of Hypnotism, New York, Wiley, 1989. 
[14] Gelder Michael, Dennis Gath and Mayor Richard. Oxford Textbook of Psychiatry. Oxford: Oxford Medical Publications, 1990.

[15] Jain K, Ashok. Clinical \& Meditative Hypnotherapy. Quantum Hypnotherapy: New Jersey, 2006.

[16] Taylor F. Kraupl. Psychopathology its causes and symptom. Sunbury-on-Thames, Middlesex: Quartermain House Ltd, 1979.

[17] Yapko, M. D. Hypnosis and the treatment of Depressions. Washington: Taylor and Francis, 2001.

[18] Beck, A. Steer, R, Kovacs, M, and Garrison B. Hopelessness and eventual suicide. A 10-year prospective study of patients hospitalised with suicidal ideation. American Journal of Psychiatry 1985; 142, 559-563.

[19] Clance P. The impostor phenomenon. New York: Bantam Books, 1985.

[20] Dingwall, J. E. Abnormal Hypnotic phenomenon (4 vols) London: Churchill, 1967.

[21] Tarlaci Sultan. Neuroquantology, Quantum Physics in the Brain. New York: Nova Science Publishers, 2014.

[22] Kirsch, I. \& Lynn, S. J. The altered state of hypnosis: Changes in the theoretical landscape. American Psychologist. 1995; (50): 846-858.

[23] Kirsch, Irvine. Hypnosis in Psychotherapy: Efficacy and mechanisms. Contemporary hypnosis 1996; (13): 109-114.

[24] Rubai AI Talal. The Hypnotic Treatment of Depression, Complementary Health Practice Review. 1999; Vol 5, No2:151-162.

[25] Nappe M Vernon. Cry the Beloved Mind: A voyage of Hope. Peanut Butter Publishing: Seattle, 1999.

[26] Sanderson Alan Lindsay. Spirit release therapy; what is it and what can it achieve?

www.rcpsych.ac.uk/college/specialinterestgroups/spirituality/p ublications.aspx 2003a

[27] Sanderson Alan Lindsay. The Case for Spirit Releasement Therapy www.rcpsych.ac.uk/college/specialinterestgroups/spirituality/p ublications.aspx $2003 b$

[28] Sanderson Alan Lindsay. The Treatment of Psychotic patients with Delusional States www.rcpsych.ac.uk/college/specialinterestgroups/spirituality/p ublications.aspx 2012

[29] Wickland, C. A. Thirty Years among the Dead. Los Angeles: Llewellyn Publications, 1974.

[30] Patrnicka Wanda. Possessed by Ghosts: Exorcisms in the 21st century. Warszwa: Centrum Publishers, 2006.

[31] McCall Kenneth. Healing the family tree. Santa Barbara: Queenship Publishing Co, 1975.

[32] Modi Shakuntala. Remarkable Healings. Charlottesville: Hampton Roads Publishing Co, 1998.

[33] Klauber. D. Robert. Modern physics and subtle realms: not mutually exclusive. Journal of scientific exploration, 2000; (4):275-279.
[34] Bryan. A Ronald. Are quarks and leptons dynamically confined in four flat extra dimensions? Nuclear Physics B $1998 ; 523,232$

[35] Bryan. A Ronald. Are the Dirac particles of the Standard Model dynamically confined states in a higher dimensional flat space? Canadian Journal of Physics 1999; 77, 197.

[36] Bryan. A Ronald. What can Elementary Particles Tell US about the World in Which We Live? Journal of scientific exploration, 2000; v14, 257-274.

[37] Antoniadis, I., Arkani-Hamed, N. Dimopoulos, S., \&Dvali, G. New dimensions at a millimetre to a Fermi and superstrings at TeV. Physics Lectures B 1998; 436, 257.

[38] Banks, T., Dine, M., \&Nelson, A. E. Constraints on theories with large extra dimensions. Journal of High Energy Physics (electronic journal), 1999; 06, 014.

[39] Smythies J. R. Minds and Higher Dimensions. Journal of the Society for Psychical Research1988; Vol. 55. No, 812.

[40] Stevenson Ian. Can we Describe mind? Research in Parapsychology. Metuchen, N. J.: The scarecrow press, Inc. p 130-142, 1980.

[41] Broad C. D. An examination of McTaggart's philosophy. Vol. 2. Cambridge University Press, 1938.

[42] Pandarakalam J. P. Triune view of mind, Letter to the editor. Christian Parapsychologist 2005; March Volume 16 No5:153154.

[43] Myers F. W. H. Human Personality and its Survival of Bodily Death. London: Facsimile Publisher, 1903/2015.

[44] Jay A. The Invisible Bodies. Victoria, BC, Canada: Trafford Publishing, 2006.

[45] Goswami Amit. The idealistic interpretation of quantum mechanics. Physics essays. vol2:385-400, 1989.

[46] Goswami Amit. Physics of the Soul. Charlottesville, VA: Hampton Roads, 2001.

[47] Haisch Bernard. The God Theory; Universes, Zero-point fields, and what's behind it all. San Francisco: Weiser books, 2006.

[48] Haisch Bernard. The Purpose-Guided Universe. New Page Books: Franklin Lakes, N. J., 2010.

[49] Pandarakalam James Paul. Beyond the Quantum Mind. www.rcpsych.ac.uk/college/specialinterestgroups/spirituality/p ublications.aspx 2010.

[50] Tart T. Charles. The End of Materialism. Oakland: New Harbinger Publications, 2009.

[51] Stokes M Douglas. On the thoughts of dust. Paranormal Review 2009; (50):p 3-6

[52] Dannet Daniel (1991) Consciousness Explained. New York: Little Brown\&Co, 1991.

[53] Chopra Deepak. How to Know God. London: Rider, 2001.

[54] Cabanac Michel, Cabanac Remi and Hammel T Harold. Universe Consciousness: The Fifth Influence, in Consciousness and the Universe. Cambridge: Cosmology Science Publishers, 2011. 
[55] Tymn Michael. Were the Las Vegas shooter and Austin bomber possessed? http://whitecrowbooks.com/michaeltymn/ Posted on 07 May 2018.

[56] Partie Rajive \& Perry Paul. Dying to Wake Up. Atria Books: New York, 2016.

[57] McAll Kenneth. Healing the Family Tree. Santa Barbara:Queenship Publishing Co, 1996.

[58] McAll Kenneth. Guide to Healing the Family Tree. Carberry: The Handsell Press Ltd. 1994.

[59] McAll Kenneth. Healing the Haunted. Hampshire: Bignell wood. 1989.

[60] Chopra Deepak. Quantum Healing. New York: Bantam, 2015.

[61] Ehrenwald, J. New Dimensions of Deep Analysis. New York: Arno Press, 1954.

[62] Ehrenwald, J. Psi and Psychoanalysis. New York: Grune\& Stratton, 1975a.

[63] Ehrenwald, J. Telepathy and Medical parapsychology. New York: Gordon Press, 1974.

[64] Erhenwald, J. History of Psychotherapy: From Healing Magic to Encounter groups. New York: Jason Aronson, 1975b.

[65] Ghaemi Nassir: Quantum Psychiatry: A new Paradigm? Journal of Neuroquantology 2010; 8:49-50.

[66] Globus Gordon: Toward Quantum Psychiatry: Hallucinations, Thought Insertion and DSM. Journal of Neuroquantology 2010; 1: 1-12.

[67] Globus Gordon: Inner Speech and the Linguistic Sign: Toward Quantum Semiology. Journal of Neuroquantology 2011; 9(2): 243-254.

[68] Zizzi Paola, Pregnolato Mussemo. Quantum logic of the Unconscious and Schizophrenia. Journal of NeuroQuantology 2012; 10:566-579.

[69] Penrose, Roger. Shadows of the mind: A search for the missing science of Consciousness. Oxford: oxford University Press, 1994

[70] Penrose, Roger et al. Consciousness and the Universe. Cambridge MA: Cosmology Science Publishers, 2015.
[71] Wasserman DG. Shadow Matter Body and Psychic Phenomenon. Oxford: Mandrake of Oxford, 1993.

[72] Pandarakalam James Paul. The Concept of the Lighter Shadow Matter Body and Brain: A Valuable Contribution to Mind-Brain Problem. NeuroQuantology 2015; 13(2):141-153.

[73] Moncrieff Joanna, Kirsch Irvine. Efficacy of antidepressants in adults. BMJ 2005: 155-157, doi: 10. 1136/bmj, 331. 7509. 155

[74] Pandarakalam James Paul. Are the Antidepressants really antidepressants? BMJ rapid response. $14^{\text {th }}$ July, 2005.

[75] Shelton RC. Treatment options of Refractory depression. J. Clinical Psychiatry 1999; 60 (suppl 4):57-61.

[76] Depression Guideline Panel. Depression in primary care, Vol2 Treatment of major depression. Clinical practice guideline 1993, No 5. pp71-86. Department of Health and Human Services. Rockville, MD.

[77] McAllister Williams RH, Christmas DMB, Cleare AJ, Currie A, Gledhill J, Insole L, et al. Multiple- therapy-resistant-major depressive disorder; a clinically important concept. Br. J. Psychiatry2018; 212: 274-278.

[78] Anderson M. Ian. We all know what we mean by treatmentresistant depression-don't we? Br. J. Psychiatry 2018; 212: 259-261.

[79] Pandarakalam James Paul. Clinical Challenges of bipolar depression. Journal of Hospital Medicine 2007; 68 (10):530537.

[80] Hirschfield RM, Calabrese JR, Weissman MM, Screening for bipolar disorder in the community. J. Clinic Psychiatry 2003; 64:53-59.

[81] Mindell Arnold. Quantum Mind; The Edge between Physics and Psychology. Oregon: Lao Tse Press, 2000.

[82] Dispenza Joe. You are the Placebo, Making Your Mind Matter. London: Hay House Publishing, 2014.

[83] Kirsch Irving. The Emperor's New Drugs, Exploding the Antidepressant Myth. London: Bodly Head. 2009. 